\title{
Case Report \\ Tuboovarian Abscess as Primary Presentation for Imperforate Hymen
}

\author{
Jeh Wen Ho, D. Angstetra, R. Loong, and T. Fleming \\ Department of Minimally Invasive Gynaecology, The Gold Coast University Hospital, Level 1B Block North, \\ 1 Hospital Boulevard, Southport, QLD 4215, Australia \\ Correspondence should be addressed to Jeh Wen Ho; jehwenho@gmail.com
}

Received 29 January 2014; Revised 17 March 2014; Accepted 24 March 2014; Published 16 April 2014

Academic Editor: Ching-Chung Liang

Copyright (C) 2014 Jeh Wen Ho et al. This is an open access article distributed under the Creative Commons Attribution License, which permits unrestricted use, distribution, and reproduction in any medium, provided the original work is properly cited.

Objective. Imperforate hymen represents the extreme in the spectrum of hymenal embryological variations. The archetypal presentation in the adolescent patient is that of cyclical abdominopelvic pain in the presence of amenorrhoea. We reported a rare event of imperforate hymen presenting as a cause of tuboovarian abscess (TOA). Case Study. A 14-year-old girl presented to the emergency department complaining of severe left iliac fossa pain. It was her first episode of heavy bleeding per vagina, and she had a history of cyclical pelvic pain. She was clinically unwell, and an external genital examination demonstrated a partially perforated hymen. A transabdominal ultrasound showed grossly dilated serpiginous fallopian tubes. The upper part of the vagina was filled with homogeneous echogenic substance. Magnetic resonance imaging (MRI) demonstrated complex right adnexa mass with bilateral pyo-haemato-salpinges, haematometra, and haematocolpos. In theatre, the imperforate hymen was opened via cruciate incision and blood was drained from the vagina. At laparoscopy, dense purulent material was evacuated prior to an incision and drainage of the persistent right TOA. Conclusion. Ideally identification of imperforate hymen should occur during neonatal examination to prevent symptomatic presentation. Our case highlights the risks of late recognition resulting in the development of sepsis and TOA.

\section{Introduction}

Imperforate hymen represents the extreme in the spectrum of embryological variations in hymenal configuration, with reported incidence ranging from $0.014 \%$ to $0.1 \%$ [1]. Antenatal diagnosis of imperforate hymen is challenging; thus, neonatal diagnosis is optimal to prevent symptoms and complications seen when primary presentation occurs in adolescence.

The archetypal presentation in the adolescent patient is that of cyclical abdominopelvic pain in the presence of amenorrhoea [2]. The accumulated blood behind an intact hymen may compress the adjacent pelvic organs or vessels, resulting in some of the less common presentations such as urinary retention, back pain, or constipation. We report a rare case of spontaneous partial rupture of an imperforate hymen resulting in tuboovarian abscess (TOA) and sepsis.

\section{Case Study}

A 14-year-old girl presented to the emergency department of the Gold Coast University Hospital complaining of severe left iliac fossa pain with her first episode of heavy bleeding per vagina since the morning of presentation. Her background history was unremarkable with no significant medical or surgical history. She had no previous sexual encounters (virgo intacta). Further questioning elucidated that she had not gone through menarche and had cyclical pelvic pain for the previous three linebreak months.

Examination revealed a clinically unwell patient. She presented as febrile to 39 degrees Celsius, with tachycardia of $118 \mathrm{bpm}$. Her blood pressure was 120/60, showing a postural drop to $105 / 40$. Her abdomen was soft; however there was marked lower abdominal tenderness on palpation, rebound tenderness and abdominal guarding. External genital examination was limited by copious amounts of both old and fresh 
Echogenic substance within endometrial cavity

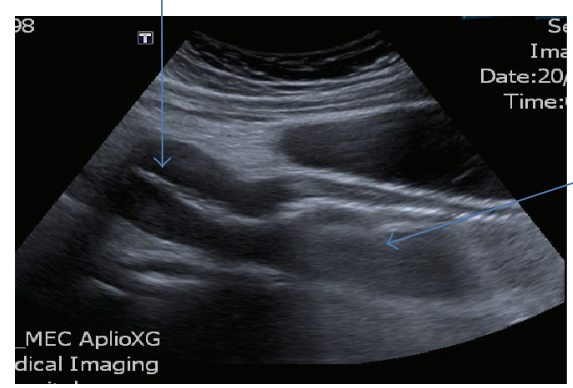

Dilated vagina filled with echogenic substance

FIgURE 1: Transabdominal ultrasound of uterus and vagina.

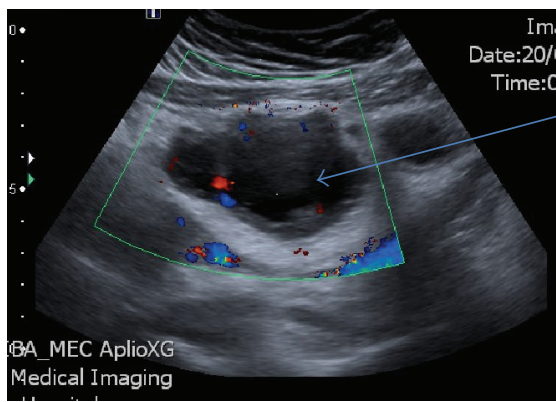

Right adnexa

complex mass

Figure 2: Transabdominal ultrasound of right adnexa-complex mass noted $70 \mathrm{~mm} \times 45 \mathrm{~mm} \times 42 \mathrm{~mm}$.

blood. However, it did reveal a partially perforated hymen with the rest of the hymen still intact. Intravenous antibiotics were empirically commenced.

Biochemical workup indicated signs of infection, with a c-reactive protein of 315 , elevated white cell count of 12 , and a neutrophil count of 8.9. Blood cultures were obtained, and ultimately a coagulase negative staphylococcus (probable skin contaminant) was grown. The urine specimen was grossly contaminated with blood and grew no organism. The beta-human chorionic gonadotropin was negative.

The transabdominal ultrasound images showed grossly dilated, serpiginous fallopian tubes with low level internal echoes and peripheral vascularity. There was echogenic substance within the endometrial cavity; the upper part of vagina was dilated and filled with homogenous echogenic substance (Figure 1). A right complex mass was noted on ultrasound (Figure 2). The lower vagina was not well visualized. The renal tract was assessed as normal. Magnetic resonance imaging (MRI) further elucidated the complex right adnexa mass with bilateral pyo-haemato-salpinges, haematometra, and haematocolpos (Figures 3 and 4). The pictures were in keeping with a partial imperforate hymen rather than vaginal septum.

An examination under anaesthesia with diagnostic laparoscopy was undertaken. The imperforate hymen was opened via a cruciate incision and blood drained from distended and oedematous upper vagina. Purulent material

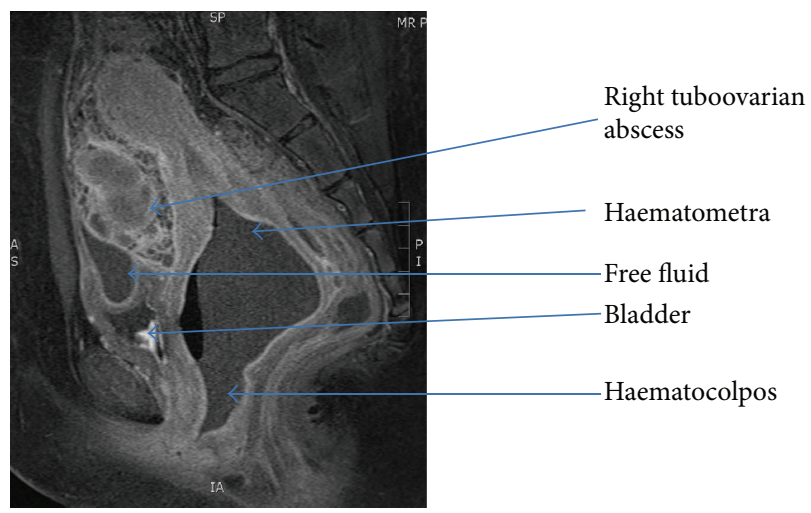

FIGURE 3: Sagittal view T1 weighted MRI abdomen/pelvis.

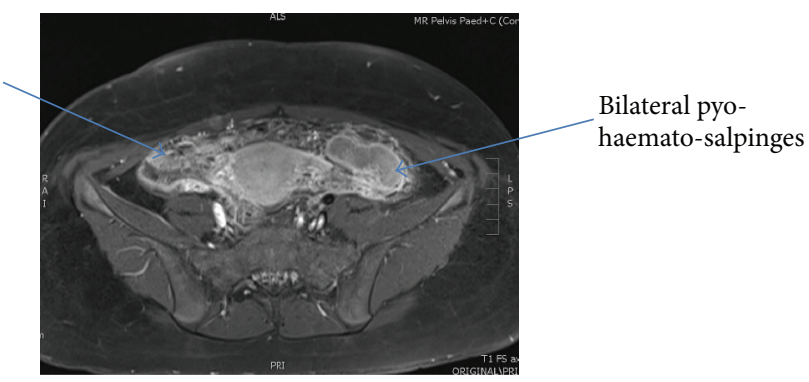

FIgURE 4: Axial view T1 weighted MRI pelvis.

in the abdominal cavity was drained, and division of omental adhesions to uterus and fallopian tubes, caecum, and appendix was necessary to adequately view pelvic organs. A right TOA was identified, incised, and drained (Figure 5). A thorough washout was performed and a pelvic drain was inserted.

She had an uncomplicated recovery and was discharged six days following the procedure with a course of oral antibiotics. At outpatient review six weeks following surgery, she reported a resolution of her dysmenorrhoea with the menstrual cycle experienced postoperatively.

\section{Discussion}

A literature search was conducted using Medline, Pubmed, and Cochrane with the terms [mullerian anomaly] OR [mullerian tract] OR [imperforate hymen] OR [vaginal septum] AND [pelvic abscess] OR [pelvic infection]. This yielded several case reports of common and unusual presentations for imperforate hymen.

On occasion, antenatal ultrasound can detect an imperforate hymen due to the presence of hydrocolpos in the fetus in response to maternal oestrogens [11]. Imperforate hymen can also be identified on examination of the newborn, and if it is asymptomatic at this time, the recommendation is to delay surgical management until puberty, when the Oestrogenization improves elasticity and healing. The diagnosis is infrequently made in infancy, if the patient presents with a mucocele, in which case hymenectomy may be indicated. 


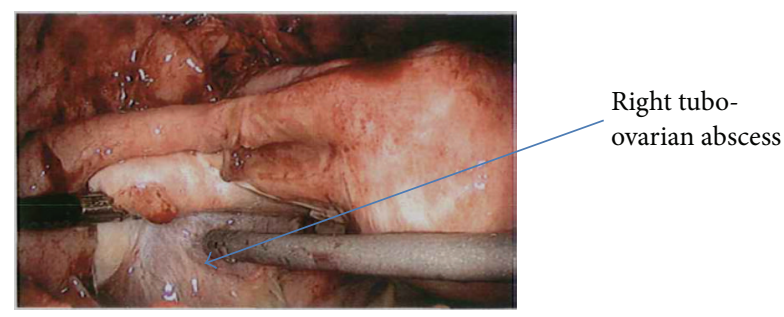

FIGURE 5: Image from diagnostic laparoscopy procedure.

TABLE 1: Unusual presentations of imperforate hymen.

\begin{tabular}{lcc}
\hline Presentation & Authors & Year \\
\hline Back pain/sciatica & Bapat and Bergsman [3] & 2008 \\
& Drakonaki et al. [4] & 2010 \\
\hline Recurrent UTI & Bursac et al. [5] & 2012 \\
\hline Iatrogenic pyocolpos & Lok and Yip [6] & 2001 \\
\hline Pelvic abscess & Sanfilippo and Mansuria [7] & 2006 \\
\hline & Dane et al. [8] & 2007 \\
Urinary retention & Ercan et al. [9] & 2011 \\
& Gyimadu et al. [10] & 2009 \\
\hline
\end{tabular}

A retrospective cohort study demonstrated a bimodal distribution of age at diagnosis. $43 \%$ of patients were diagnosed at less than 4 years of age, and the remaining 57\% were diagnosed over 10 years of age. In the group of patients diagnosed over 10 years, $100 \%$ of patients were asymptomatic, compared with just $10 \%$ of girls diagnosed under the age of 4 years [2].

In spite of recommendations for early inspection of the external genitalia, variations in hymenal anatomy frequently escape diagnosis until menarche. Although the most common adolescent presentation of an imperforate hymen is haematocolpos manifesting as abdominal pain [2], there are several case reports of more unusual or severe presentation of imperforate hymen, and these are presented in Table 1.

Of interest are the two case reports of pelvic infection arising from an imperforate hymen, which demonstrates the breakdown of the barrier from a sterile haematocolpos, either iatrogenically [6], or embryologically [7]. This paralleled our case presentation in that our patient did not become septic until the spontaneous rupture of the hymen which thus facilitated ascending infection of her reproductive tract.

Only one paper reviewed the long term outcome of late diagnoses of imperforate hymen. It reported the persistence of menstrual dysfunction even after hymenectomy, with $60 \%$ demonstrating abnormal menstruation and $40 \%$ with ongoing dysmenorrhea [11]. Furthermore, the data assessing fertility in these patients is sparse but encouraging. $86 \%$ of women who attempted pregnancy conceived after surgical correction of their imperforate hymen [12].

\section{Conclusion}

Ideally identification of the imperforate hymen should occur during the neonatal examination. Delay in diagnosis results in symptomatic presentations, with the potential for longterm menstrual and reproductive ramifications. Our case highlights the risks of late recognition of imperforate hymen, through the development of sepsis and TOA.

\section{Conflict of Interests}

The authors declare that there is no conflict of interests regarding the publication of this paper.

\section{References}

[1] A. S. Eksioglu, H. A. Maden, G. Cinar, and Y. T. Yildiz, "Imperforate hymen causing bilateral hydroureteronephrosis in an infant with bicornuate uterus," Case Reports in Urology, vol. 2012, Article ID 102683, 4 pages, 2012.

[2] J. C. Posner and P. R. Spandorfer, "Early detection of imperforate hymen prevents morbidity from delays in diagnosis," Pediatrics, vol. 115, no. 4, pp. 1008-1012, 2005.

[3] R. Bapat and C. Bergsman, "Hematometrocolpos presenting as sciatica, constipation, and urinary retention," Clinical Pediatrics, vol. 47, no. 1, pp. 71-73, 2008.

[4] E. E. Drakonaki, I. Tritou, G. Pitsoulis, K. Psaras, and E. Sfakianaki, "Hematocolpometra due to an imperforate hymen presenting with back pain: sonographic diagnosis," Journal of Ultrasound in Medicine, vol. 29, no. 2, pp. 321-322, 2010.

[5] D. Bursac, Z. Duic, J. Z. Partl, J. Valetic, and S. Stasenko, "Hematocolpos resulting from an imperforated hymen diagnosed by ultrasound in a patient with recurrent urinary tract infections," Journal of Pediatric \& Adolescent Gynecology, vol. 25, no. 5, pp. 340-341, 2012.

[6] I. H. Lok and S.-K. Yip, "Iatrogenic pyocolpos in a young girl with imperforate hymen," Australian and New Zealand Journal of Obstetrics and Gynaecology, vol. 41, no. 1, pp. 104-105, 2001.

[7] A. M. Sanfilippo and S. M. Mansuria, "Microperforate hymen resulting in pelvic abscess," Journal of Pediatric and Adolescent Gynecology, vol. 19, no. 2, pp. 95-98, 2006.

[8] C. Dane, B. Dane, M. Erginbas, and A. Cetin, "Imperforate hymen - a rare cause of abdominal pain: two cases and review of the literature," Journal of Pediatric and Adolescent Gynecology, vol. 20, no. 4, pp. 245-247, 2007.

[9] C. M. Ercan, K. E. Karasahin, I. Alanbay, M. Ulubay, and I. Baser, "Imperforate hymen causing hematocolpos and acute urinary retention in an adolescent girl," Taiwanese Journal of Obstetrics and Gynecology, vol. 50, no. 1, pp. 118-120, 2011.

[10] A. Gyimadu, B. Sayal, S. Guven, and G. S. Gunalp, "Hematocolpos causing severe urinary retention in an adolescent girl with imperforate hymen: an uncommon presentation," Archives of Gynecology and Obstetrics, vol. 280, no. 3, pp. 461-463, 2009.

[11] C.-C. Liang, S.-D. Chang, and Y.-K. Soong, "Long-term followup of women who underwent surgical correction for imperforate hymen," Archives of Gynecology and Obstetrics, vol. 269, no. 1, pp. 5-8, 2003.

[12] J. A. Rock, H. A. Zacur, A. M. Dlugi, H. W. Jones Jr., and R. W. TeLinde, "Pregnancy success following surgical correction of imperforate hymen and complete transverse vaginal septum," Obstetrics and Gynecology, vol. 59, no. 4, pp. 448-451, 1982. 


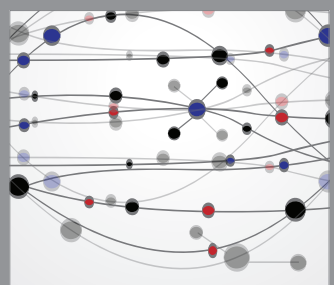

The Scientific World Journal
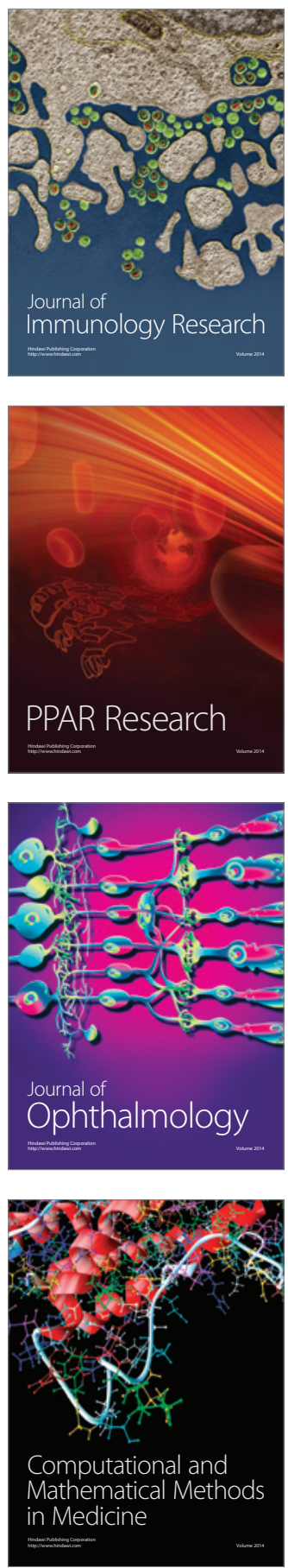

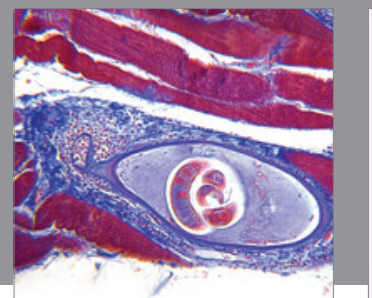

Gastroenterology

Research and Practice
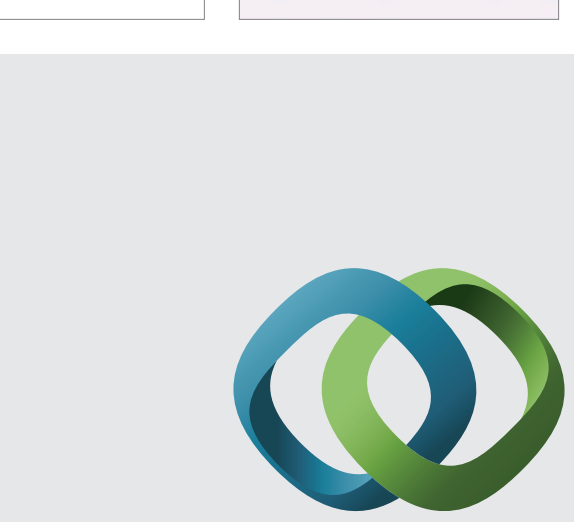

\section{Hindawi}

Submit your manuscripts at

http://www.hindawi.com
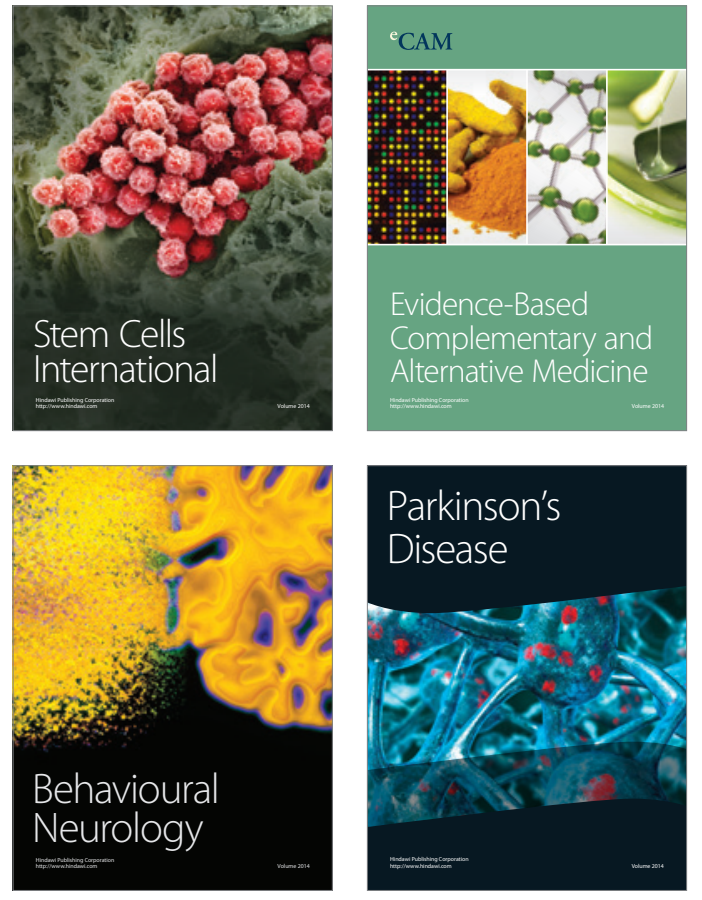
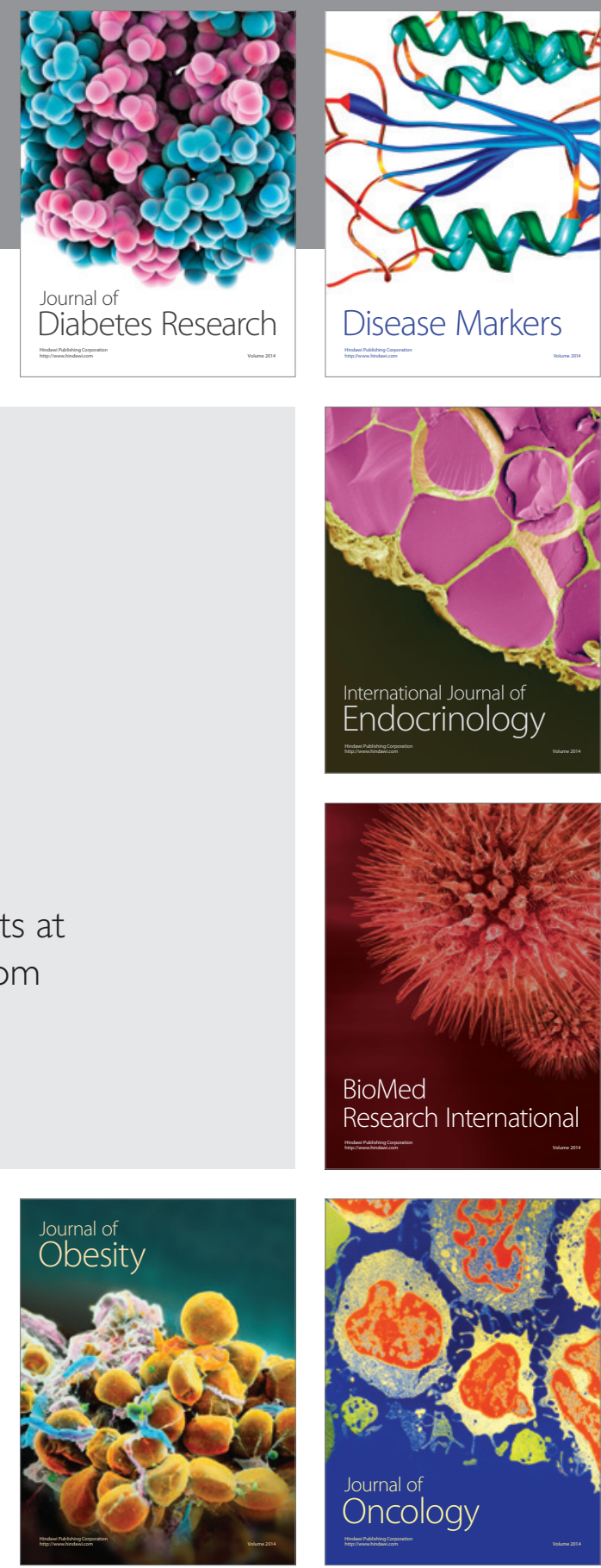

Disease Markers
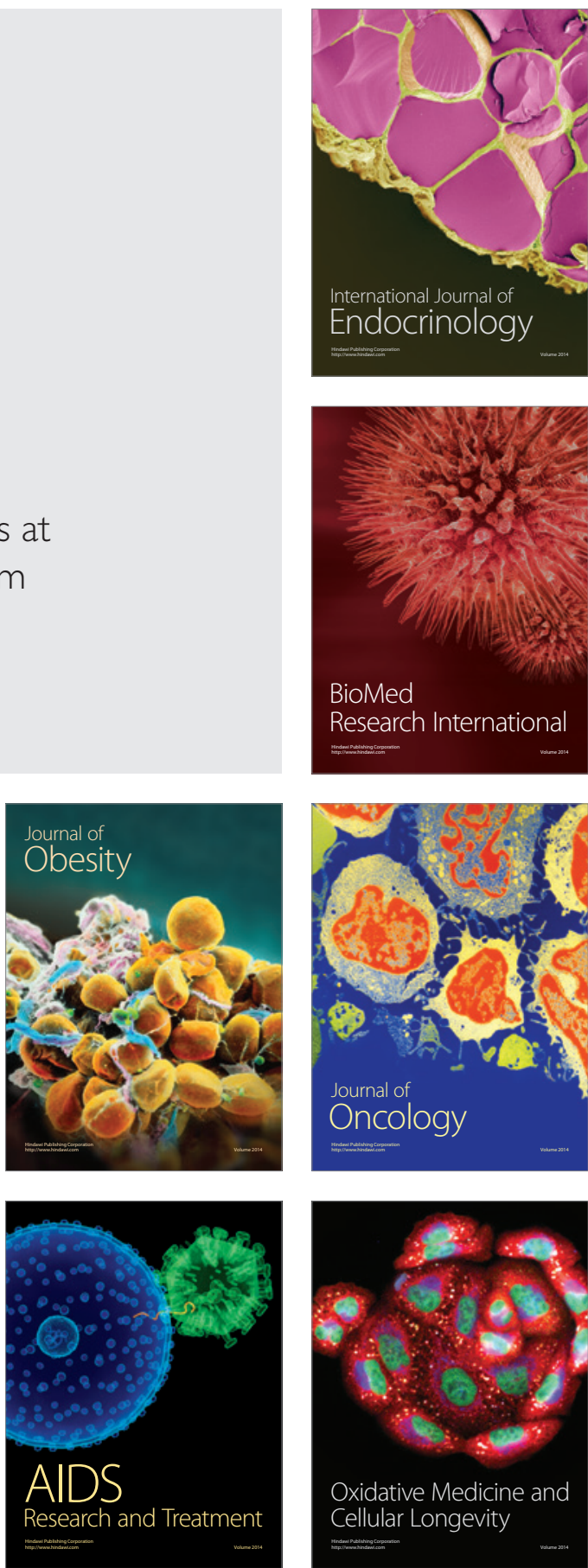